\title{
ACTIVE REGIONS
}

\author{
II : Mount Wilson 16997 \\ A Small Spot with Big Flares*
}

H. ZIRIN

The Hale Observatories Carnegie Institution of Washington, California Institute of Technology

(Received 15 April, 1969)

\begin{abstract}
We describe the great activity associated with Mount Wilson 16997, a single $\alpha p$ spot without plage that produced a number of large flares and developed into a substantial center. The activity was signalled by the emergence of $f$ polarity and weak plage ahead of the spot on 24 September 1968 (although the region was already seen at the limb at the 22 September 1968 eclipse as a active center). A number of explosive flares with X-rays on the 25 th were climaxed by a large flare a 0036 UT 26 September; the latter was associated with the splitting of the old spot and the emergence of a new one. The polarity axis rotated around to a normal configuration by the 29 th, but a large flare occurred on that day, too.

Several of the flares show interesting details such as the return of spray material to the surface at the boundary of the region.

The behavior of this region shows that:

(1) Large flares may come from small, round spots;

(2) The magnetic field rotation due to the action of the Hale-Nicholson force is a possible source of flare energy; and

(3) Appearance and disappearance of spots is clearly connected with flare activity.
\end{abstract}

\section{Introduction}

Mount Wilson 16997 is quite different from any other region studied in this series. It first appeared as a small, undistinguished-looking $\alpha p$ spot ahead of a complex but inactive region 16999. However, 16999 had a large preceding spot following it, indicating possible instability.

Great activity began following the growth of $f$ polarity preceding 16997 on 24 September 1968. A number of large flares were connected with the rotation of magnetic axis during subsequent days. On 25 September, large flares occurred with the emergence of a new sunspot, and the splitting and disappearance of an old one. The group evolved into a more stable configuration which, implausibly, produced a very large flare on 29 September.

Our data consists of cinematographic observations with the Caltech and Tel Aviv Photoheliographs, supplemented by Mount Wilson white light data and magnetograms from 22 September to 1 October. Table I shows the photoheliograph observing times; coverage was nearly continuous in the critical period of 25-27 September.

The behavior of this sunspot group shows (1) flares do not necessarily come from

* This research was supported by the National Aeronautics and Space Administration under Grant NASA NGR 05002 034, and by NSF under Grant 1472. 
TABLE I

The periods of observation

\begin{tabular}{|c|c|c|c|c|}
\hline \multirow[t]{2}{*}{ Date } & \multicolumn{2}{|c|}{ Tel Aviv } & \multicolumn{2}{|c|}{ Caltech } \\
\hline & Start & Stop & Start & Stop \\
\hline $09-22-68$ & & & $14: 41$ & $01: 20$ \\
\hline $09-23-68$ & & & $15: 38$ & $01: 26$ \\
\hline $09-24-68$ & & & $14: 47$ & $01: 28$ \\
\hline $09-25-68$ & $8: 17$ & $15: 00$ & $14: 49$ & $01: 02$ \\
\hline $09-26-68$ & $4: 17$ & $14: 58$ & $14: 43$ & $00: 44$ \\
\hline $09-27-68$ & $4: 49$ & $9: 48$ & $18: 29$ & $00: 19$ \\
\hline $09-28-68$ & & & $18: 28$ & $23: 35$ \\
\hline $09-29-68$ & $8: 29$ & $14: 35$ & & \\
\hline $09-30-68$ & $7: 37$ & $14: 25$ & & \\
\hline $10-01-68$ & & & & \\
\hline $10-02-68$ & & & $21: 26$ & $22: 03$ \\
\hline
\end{tabular}

large sunspots, but those with peculiar polarity distributions; (2) the magnetic field rotation, due to the action of the Hale-Nicholson force, is a likely source of flare energy; and (3) appearance and disappearance of new sunspots is closely connected with flare activity.

Incidentally, this region was connected with a strong coronal condensation observed on the northeast limb at the total eclipse of 22 September 1968.

\section{History of Mount Wilson 16997}

Table I gives the periods of observation with the Caltech and Tel Aviv Photoheliographs. Most of these observations were centerline, although some were off-band. Figures 1 and 2 give sequences in the development of the sunspot between 24 and 28 September, and a sequence of white light pictures is given in Figure 3. The spot was first seen on the limb on 22 September, accompanied by a small flare at 2156 UT and 0031 on 23 September. Because of the surface roughness, it is difficult to see much detail. On 23 September, it appeared as a single spot with no plage, followed by the rest of McMath Plage Region No. 9687, in which it was included. Plage No. 9687 was, in fact, the fourth appearance of a group which began as McMath 9499 (first seen 2 July) and repeated as 9564 and 9630 . Plage 9499 was the preceding part of a large complex of activity which gave many flares in July. The plage was very weak on the 7 August transit as 9564 , but experienced a rebirth of activity.

As may be seen in Figure 1, Mount Wilson 16997 looked quite undistinguished on 24 September. It was listed as $\alpha p$. and is only included in our observations because it was near other large, bright plages. There was considerable development of new active regions during this period, particularly preceding 16997 . The development of one BRL may be seen at upper right in Figure 1. However, the cinematograms for 24 September show the appearance of a region of $f$ polarity (which may be distinguished by the existence of bright $\mathrm{H} \alpha$ plage) to the northeast of the spot, accompanied 


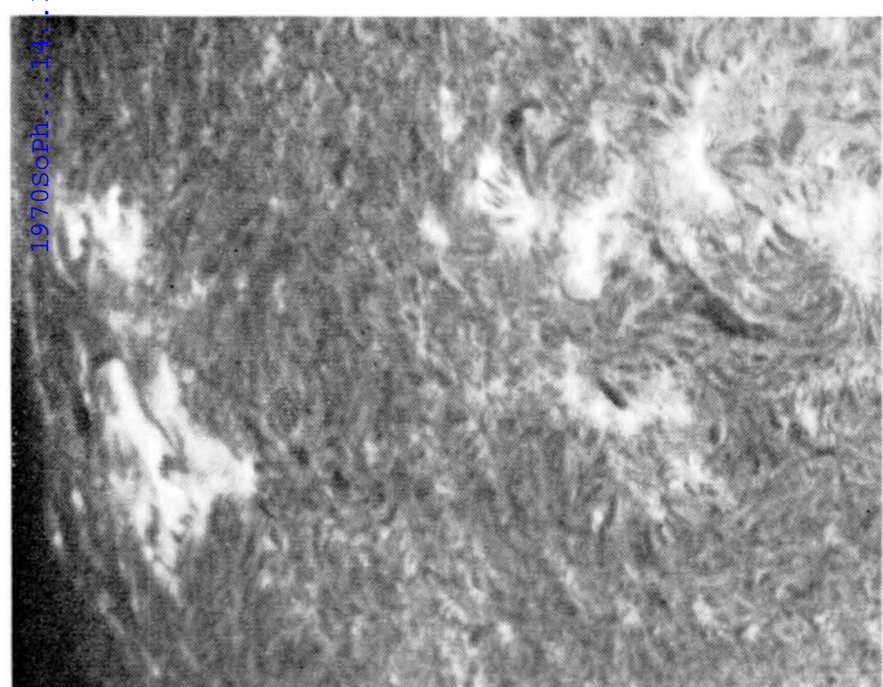

24 Sept. 0003:45

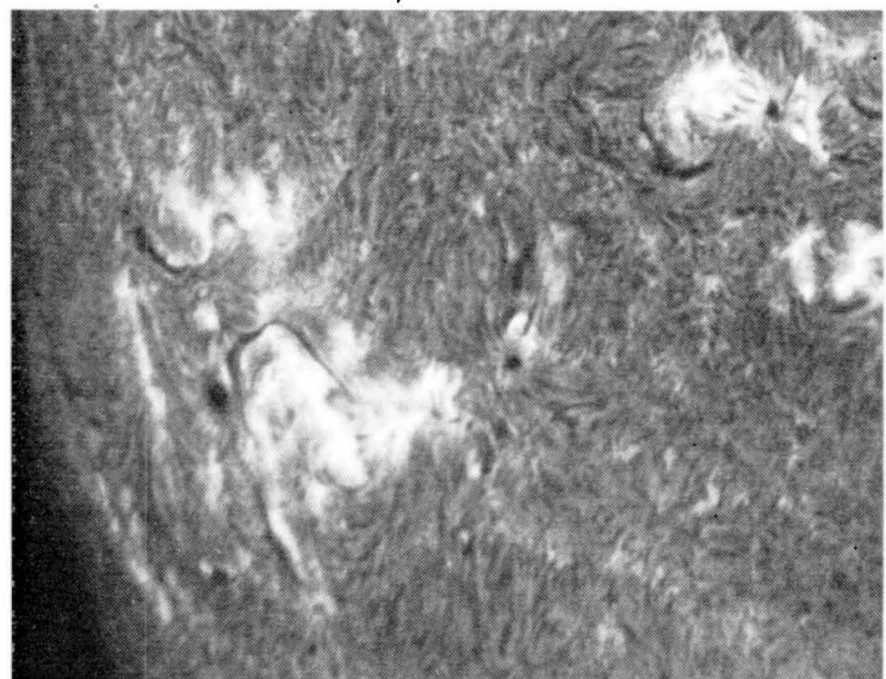

24 Sept. 2309:45

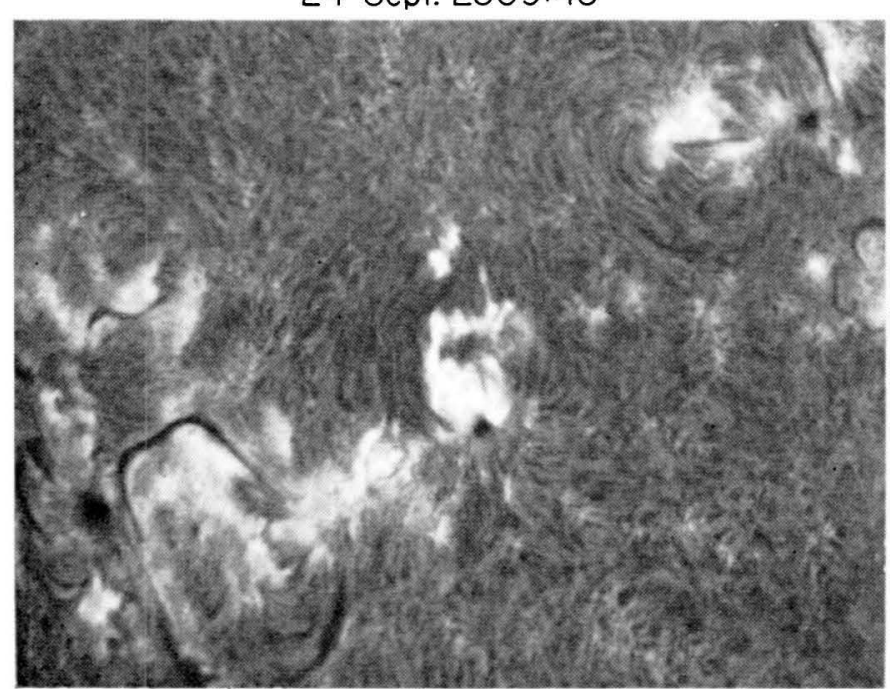

25 Sept. $1645: 20$

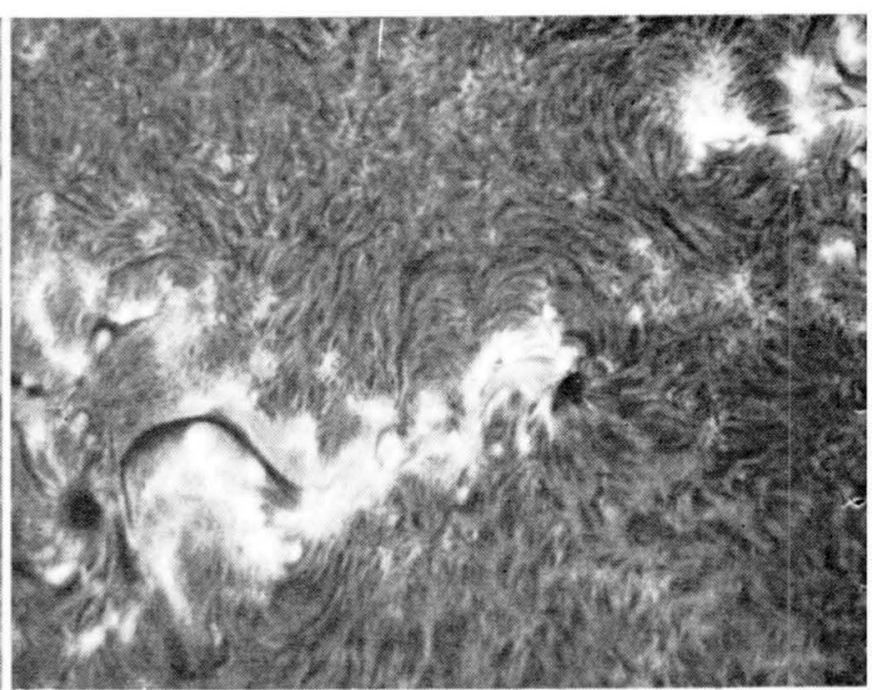

26 Sept. 1823:25

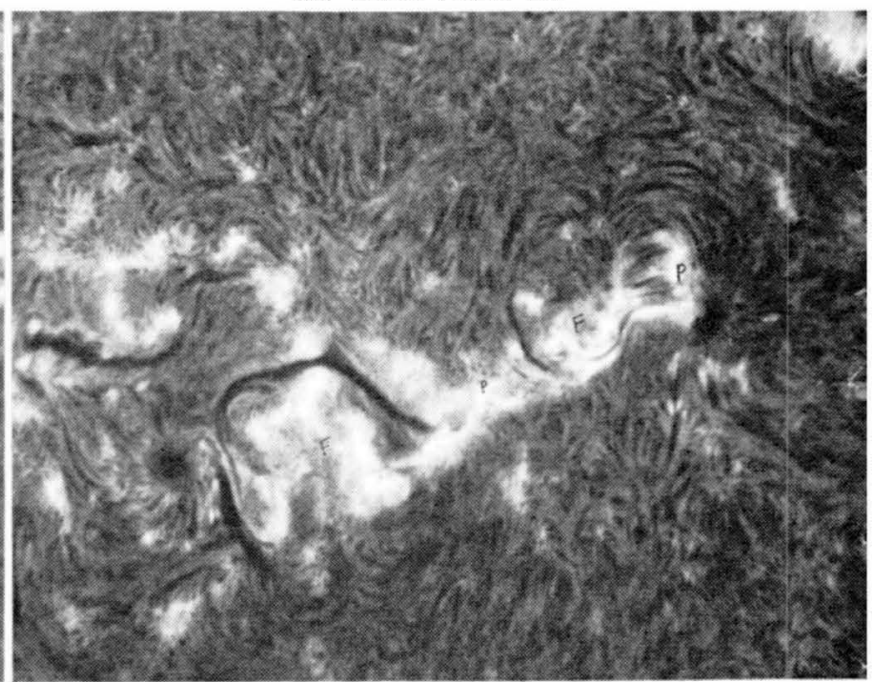

27 Sept. 1843.25

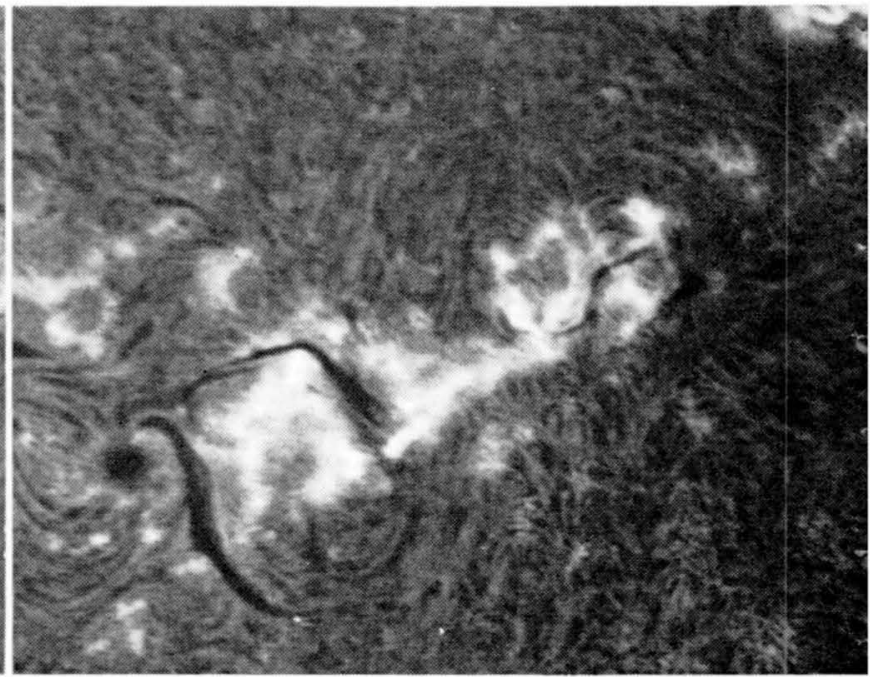

28 Sept. $1834: 42$

Fig. 1. Sunspot development 24-28 September 1968. H $\alpha$ Center.(All photos are Caltech Photoheliograph. North top, West right, unless otherwise specified). 

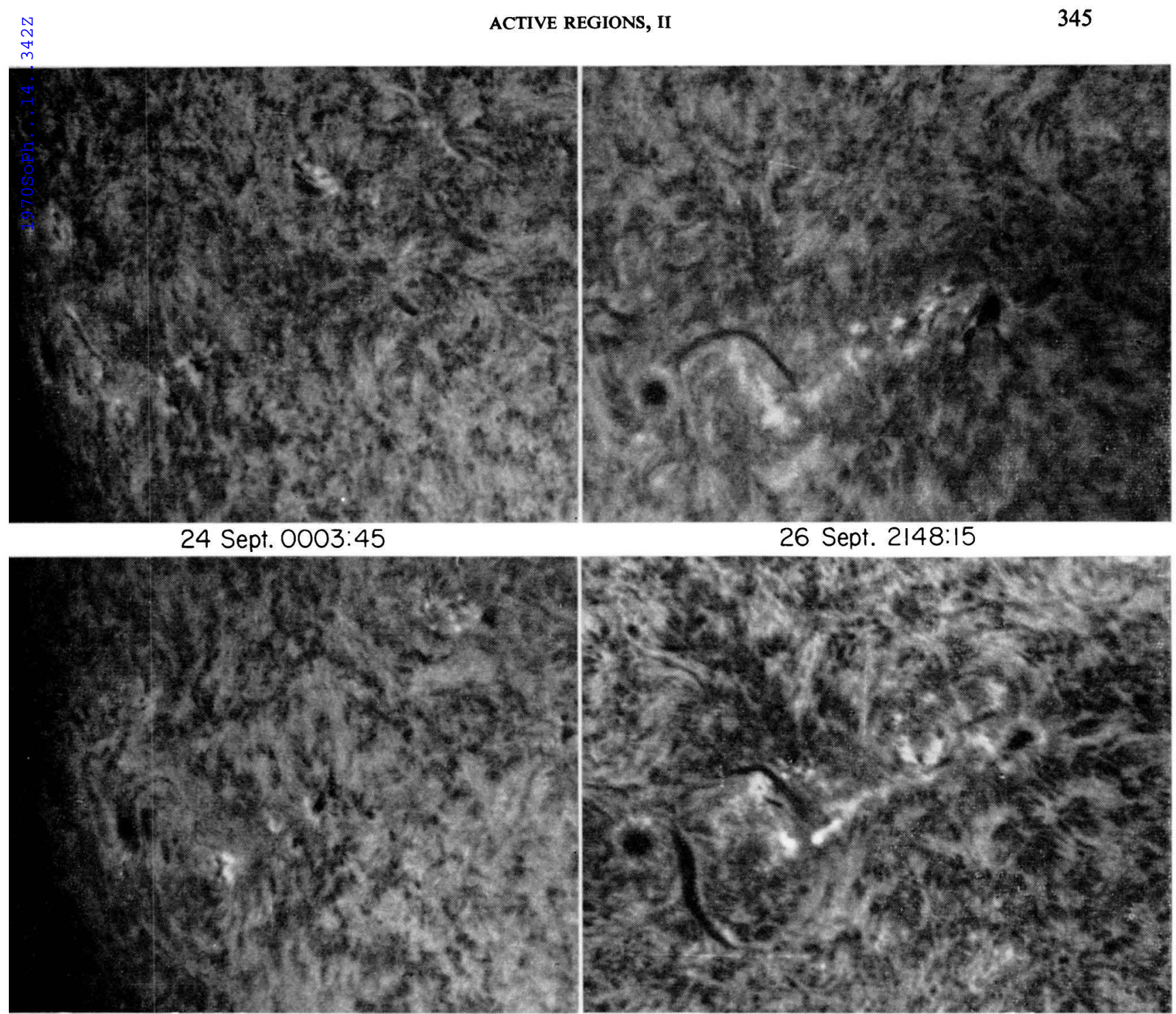

24 Sept. 1535:17

\section{Sept. $1841: 58$}

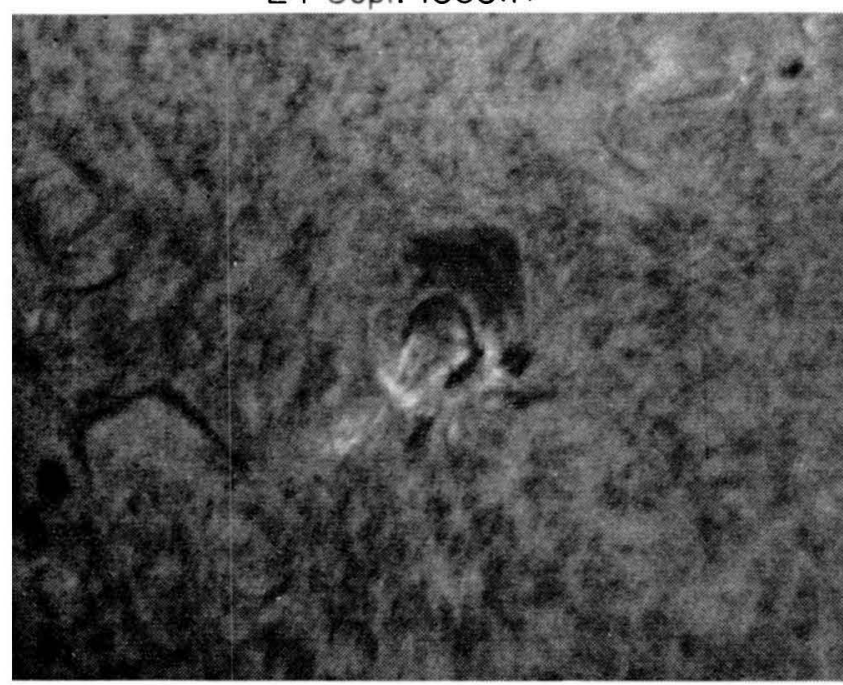

25 Sept. 2249:10

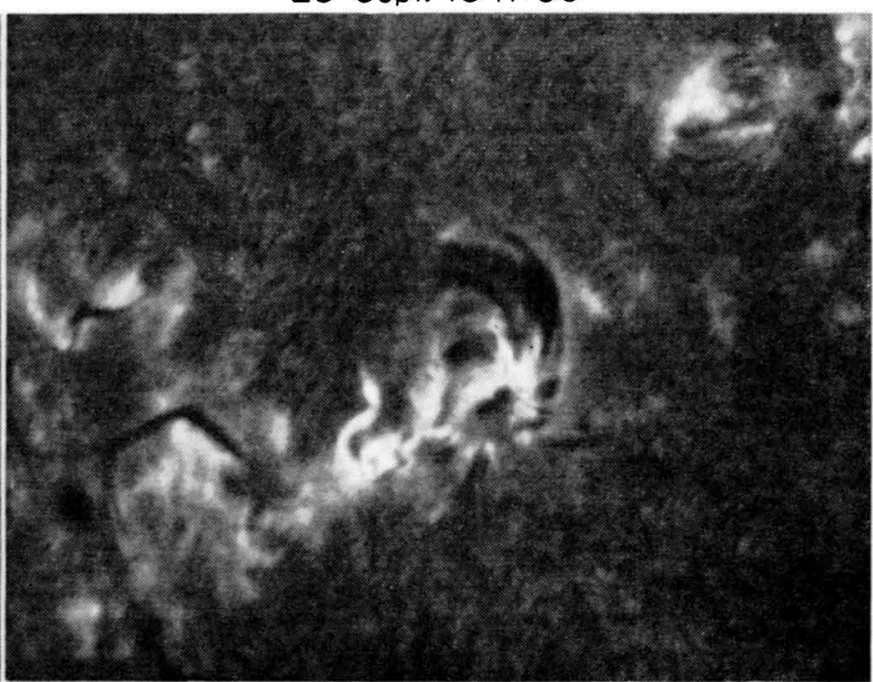

26 Sept. 0000:20

Fig. 2. Sunspot development 24-28 September 1968. H $\alpha-0.5 \AA$. 


\section{DEVELOPMENT OF MT. WILSON 16997}
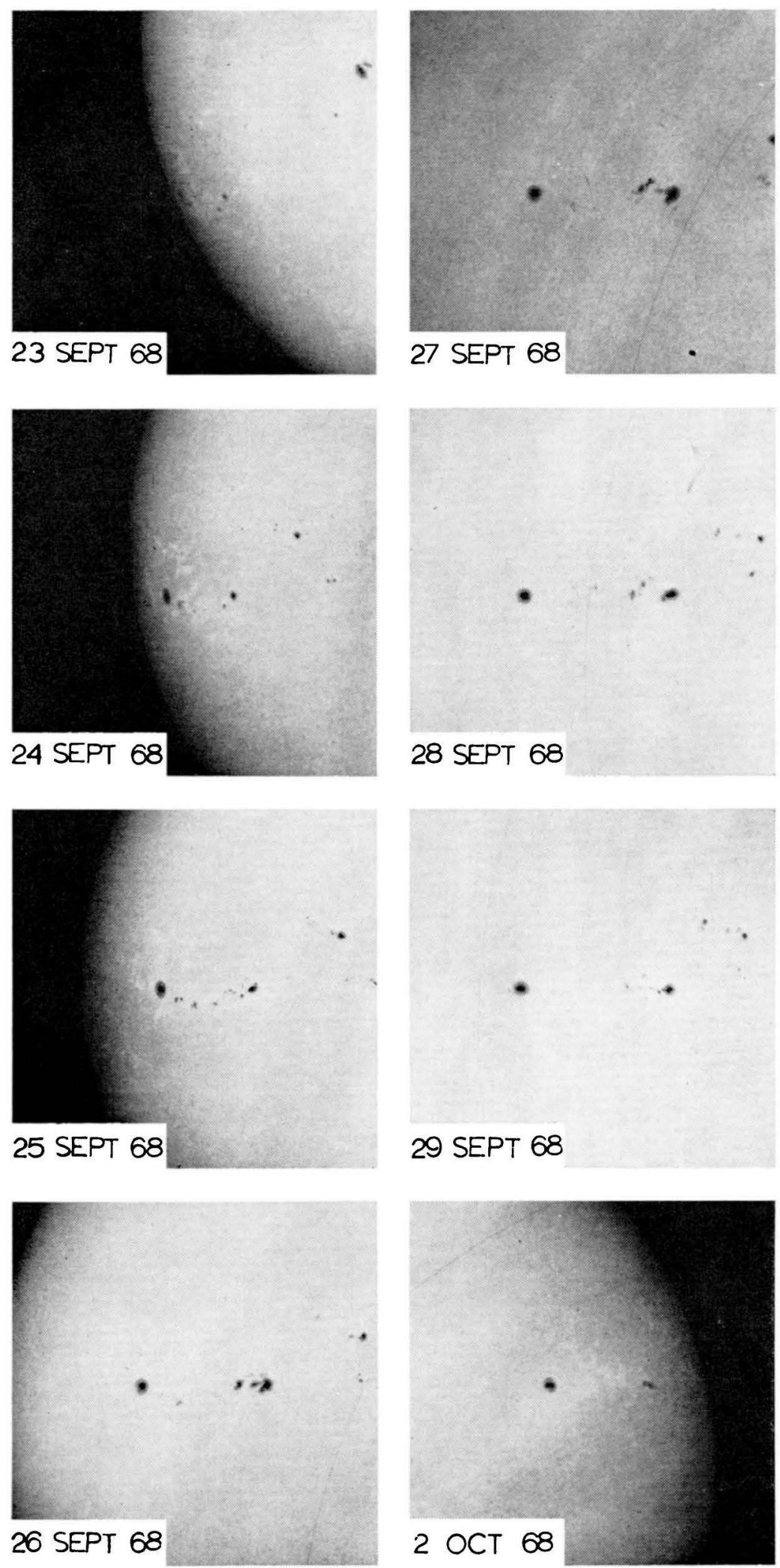

Fig. 3. White light photos, 28 September to 2 October made with the Mount Wilson 60 -foot tower. There were no observations on the 30th, but decay seems to have begun then. 
by a number of surges in that direction. During the succeeding days, great activity occurred in this region of inverted polarity as it gradually rotated until it was following the spot. Between 24 and 27 September, the rotation of a line connecting and $f$ polarity is about $110^{\circ}$. On 25 September, activity increased markedly, with a number of Class 1 explosive flares, each of which was accompanied by substantial $\mathrm{X}$-ray bursts. Figure 4 shows a series of frames taken on 25 September. In the first two frames, we may also see two bright regions with loops (BRL); one at the extreme right, and one in the middle right, marking the emergence of new sunspot fields.

The explosive flares in 16997 move from sunspot outwards in the direction of $f$ polarity. At this time, though this spot was the most active, it was relatively undistinguished in size and accompanied by only a small plage. It had the distinguishing characteristics that it was a small, round spot preceded by $f$ polarity.

Our films for 25 September are hampered by the fact that about four hours were fogged during development by the processing company (the only time this has happened), but the spot can be seen on the fogged film. About 1900 UT, it seemed to grow in elongation, so we cannot say definitely if it split or if a new spot grew alongside it. Furthermore, a third spot began to grow to the east of this point. All three spots are easily seen on the third frame of Figure 2 and the third frame of Figure 4. As these spots appeared, great loops of material rose into the atmosphere about the spot group. These loops are both dark and bright. Rapid growth can be seen from the change between frames 3 and 4 of Figure 4 . A small pre-flare occurred following the group at $00: 10: 35$ on 26 September. (Of course, we know from previous experience there is a strong probability of large flares occurring near $0 \mathrm{~h} \mathrm{UT}$ ). This was followed by a very large flare beginning at 0025 UT. The flaring developed gradually with an explosive phase around $0300 \mathrm{UT}$, which blew off all of the material on the sunspot and produced a moreton wave to the north. A second flare occurred at 00:57:45, coming from preceding spot.

Following sunset in passage, observations began at 0415 UT in Tel Aviv and showed two new flares from the new sunspot. In each case there was a rapid outward motion of material, which was suddenly deflected by the magnetic field and hit the surface in the plages to the north (this is illustrated in Figure 5). These important flares, at 0508 UT and 0846 UT, both produced moderate X-ray and radio bursts.

After 1300 UT, the old pair of spots broke up; the original spot of 16997 was no more, and the large preceding spot dominated. In fact, the three spots now in the plage of the old one were of $f$ polarity.

In this new phase of development, there was rapid growth of a following spot, accompanied by a general bright loop configuration. A number of modest flares occurred in the region. One interesting flare, at 2127 UT on the 26th, occurred simultaneously on both branches of the arched loop leading from preceding to following spots, and appears to have been entirely elevated above the chromosphere. On the 27 th, most of the flares were bright ejections from the southern edge of the penumbra of the preceding spot. There is no brightening in the umbra and penumbra, only outward spread of brightness (and material flow from the perimeter of the penumbra). 


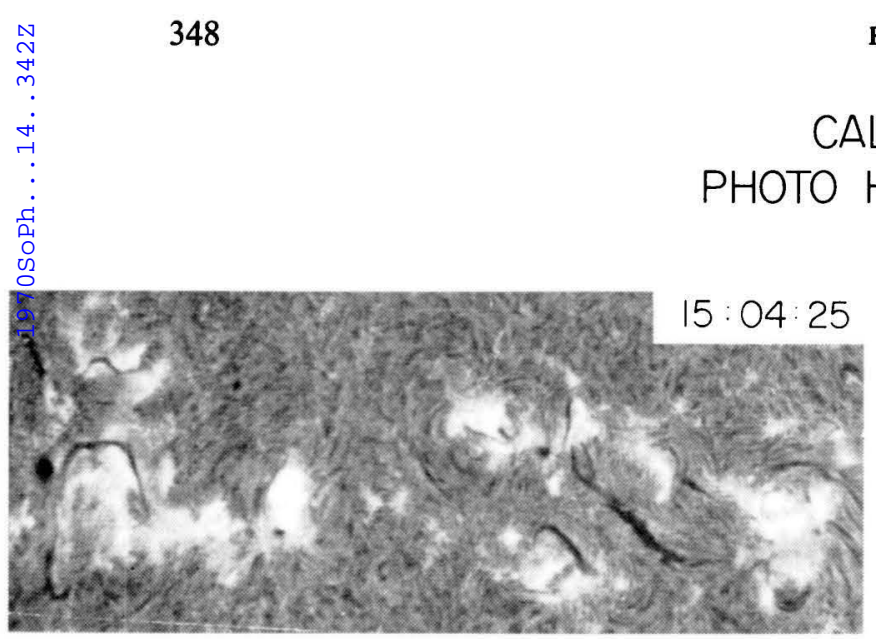

H. ZIRIN

CAL TECH

HELIOGRAPH
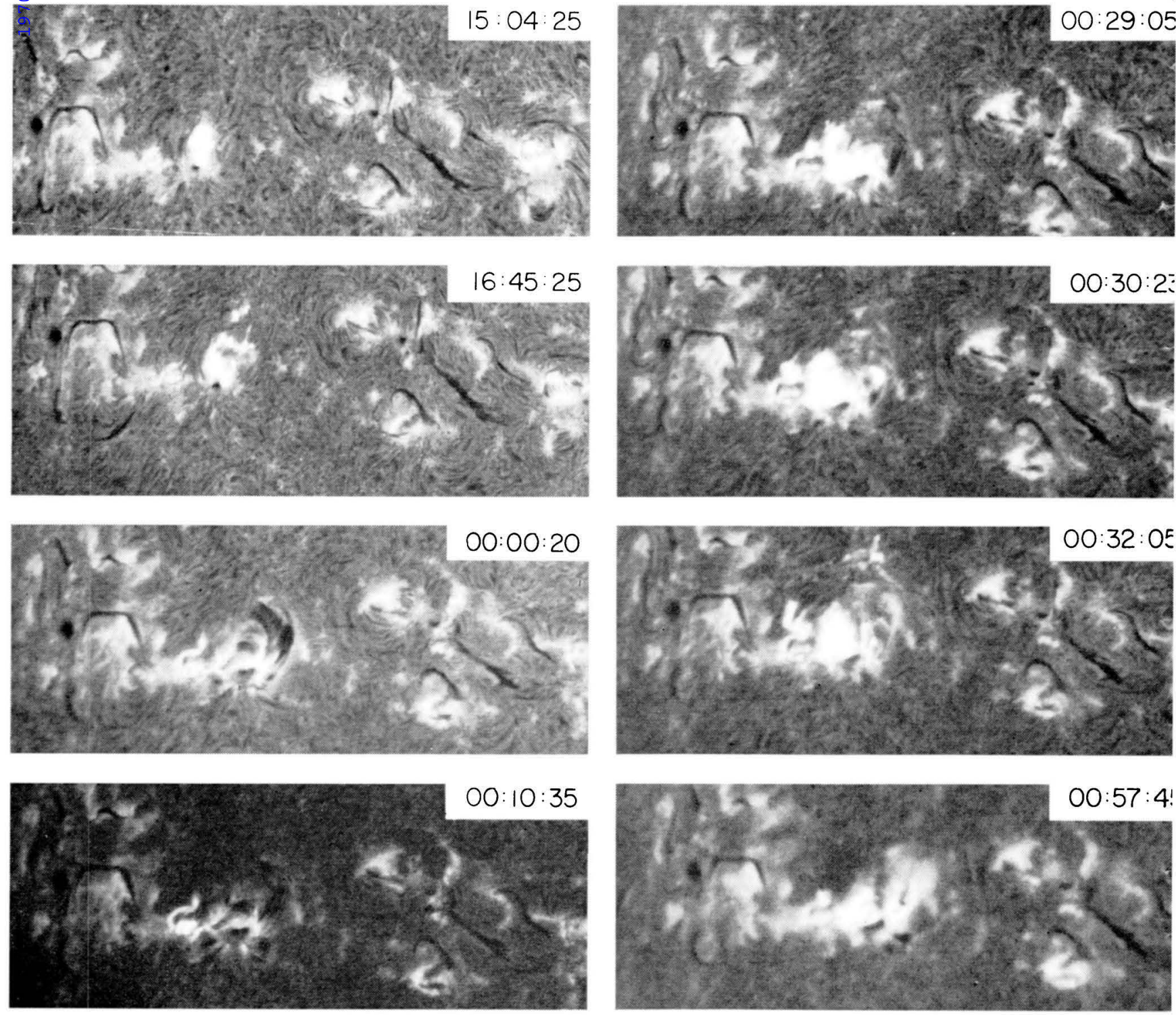

Fig. 4. A sequence of frames taken 25-26 September, showing important flares earlier in the day, and development of the large flare at 0029 (precursor at 00:10:35). Note the brightening at the top of the frame at 00:32:05, produced by excitation folling out in the flare. The early frames show development of BRL's preceding. The emergence of new spots in 16997 was accompanied by beautiful loop development beginning around 2000. These are seen in the 00:00:20 frame. 

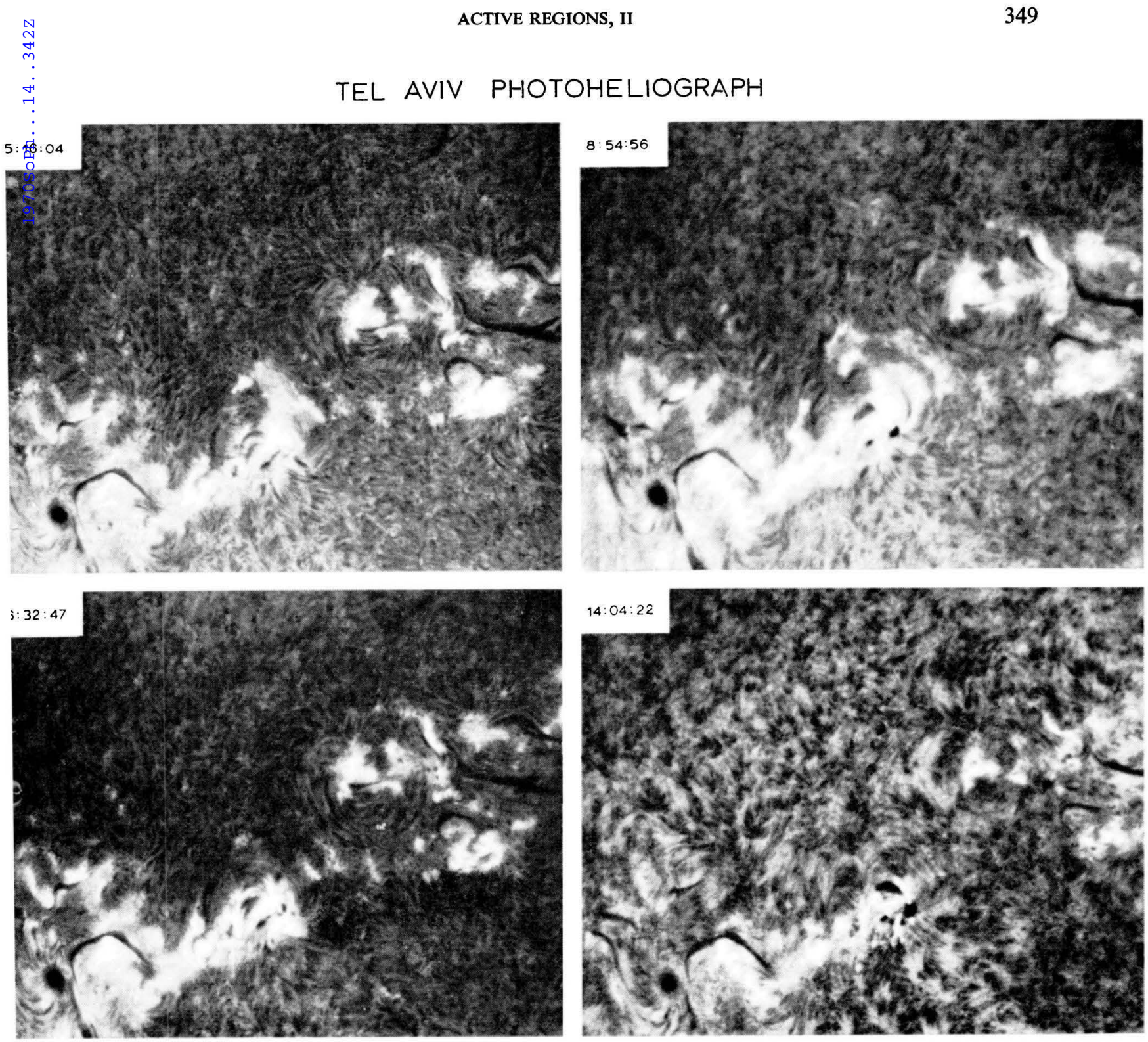

\section{SEPTEMBER 1968}

Fig. 5. Flare activity photographed at Tel Aviv, 26 September.

(A) 05:16:04, explosive flare with material crashing to the surface north of spot.

(B) $06: 32: 47$, smaller flare south of the developing spot.

(C) Repeat of A at 08:54:56, again unable to penetrate the magnetic field and crashing to the surface.

(D) 14:04:22, showing great increase in size of new spot.

At 0153 UT 28 September, a large flare was reported by several stations; we do not have pictures, but the position appears to have been north of the spot, in the area of bright loop development.

On 28 September, Figures 1 and 2 show the bright loop development had ceased and a neutral line marked by a prominence had developed. There still was bright plage between the preceding spot and the filament; normally this would indicate $f$ polarity, but the present case must either be an exception or a very complicated configuration. 


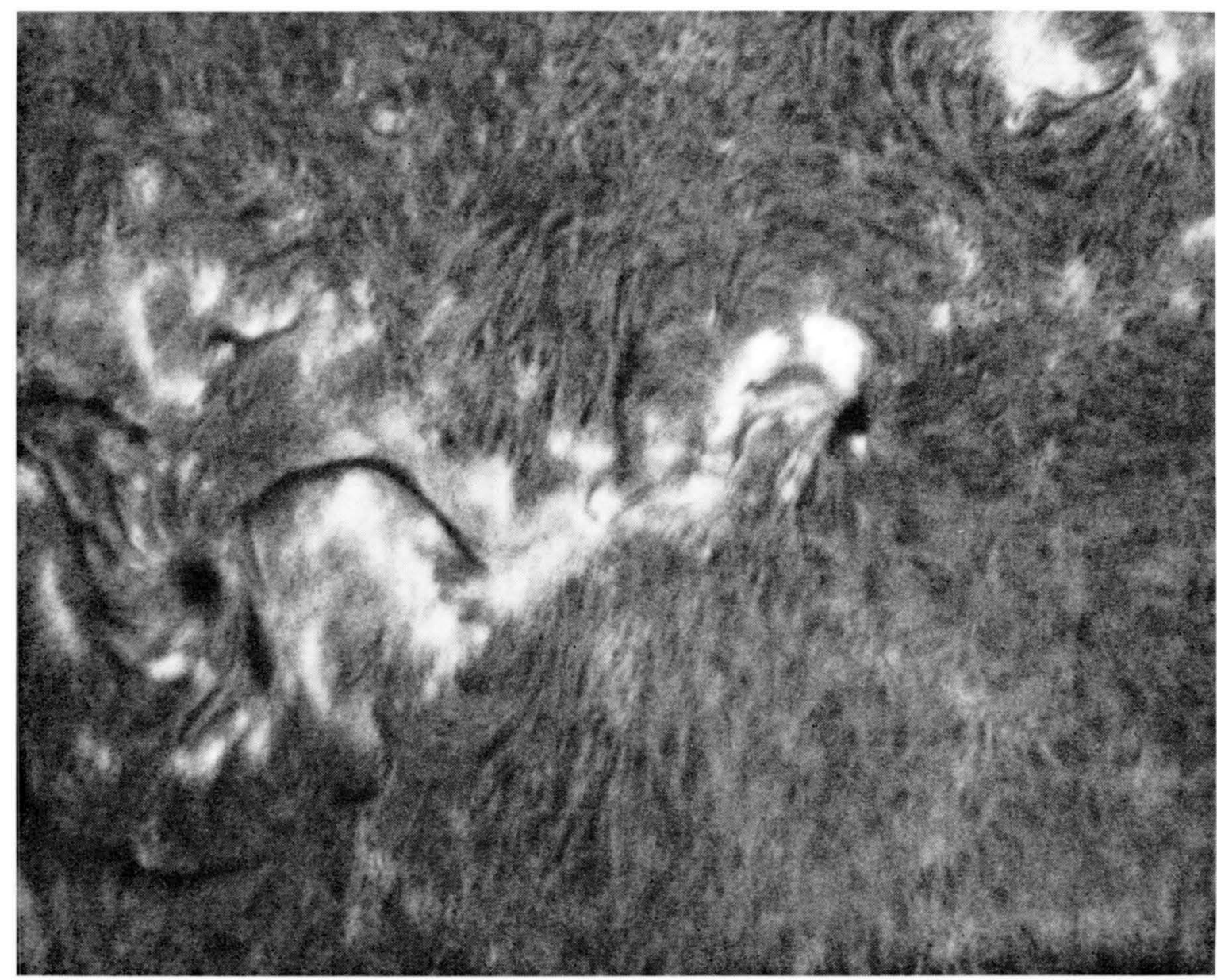

Fig. 6. Flare on both sides of the arch along emerging flux loops, 2127 UT 26 September 1968.

Thus, the situation on the 29th was fairly stable, except that there were large preceding spots at both ends of the huge group, and considerable polarity change in between. We have marked these in Figure 1, 27 September. They were marked by the filaments and the plages. Out in front is the preceding spot of Mount Wilson 16997; behind it a filament marking the polarity change to the $f$ polarity region that has been growing since the $24 \mathrm{th}$, and now rotated around to follow the spot. Behind this is a large area of $p$ polarity, which does not show the 'anti-plage' characteristics because there is no $p$ spot (compare with the $p$ spot following). Then there is a large $f$ plage surrounded by the big filament, and, bringing up the rear, the big $p$ spot, surrounded by anti-plage, which showed no activity until the 29 th.

There was little to suggest that a very large flare should occur on this day. The plage brightness was greatly reduced and no new field was emerging. Some of the filaments had changed, but changes of this type have not hitherto been connected with large flares.

Despite this fact, a large flare and several modest ones occurred in three hours, as illustrated in Figure 7 (another large flare in Plage 9678 occurred at 1618 UT but was presumably unrelated). We are puzzled by this occurrence and hope that further study will reveal the reason for this occurrence. 


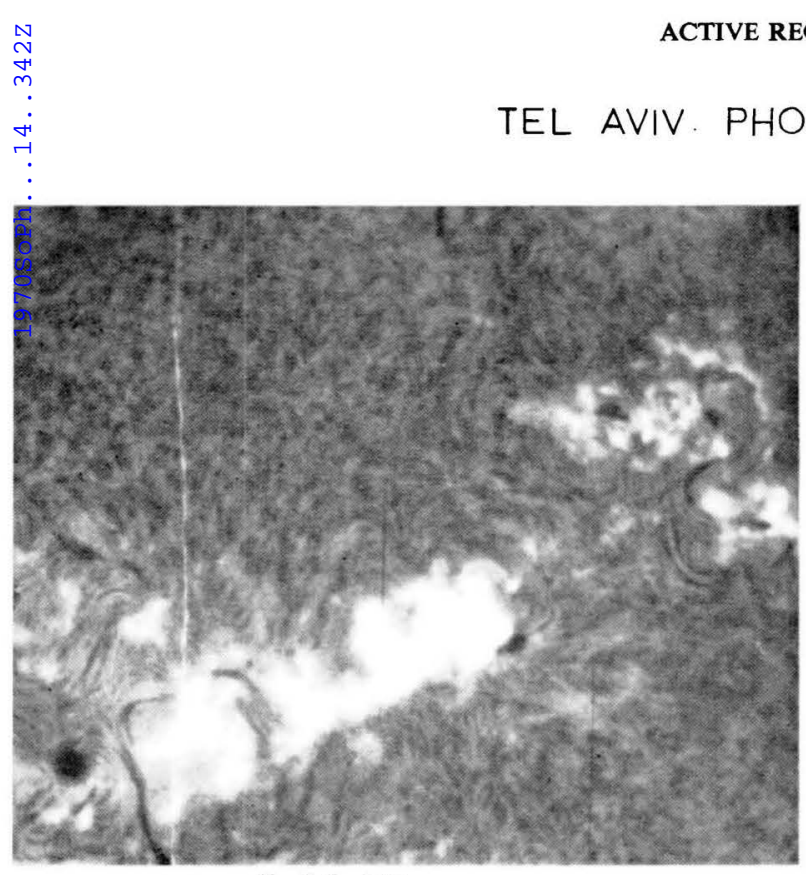

$9: 28: 37$

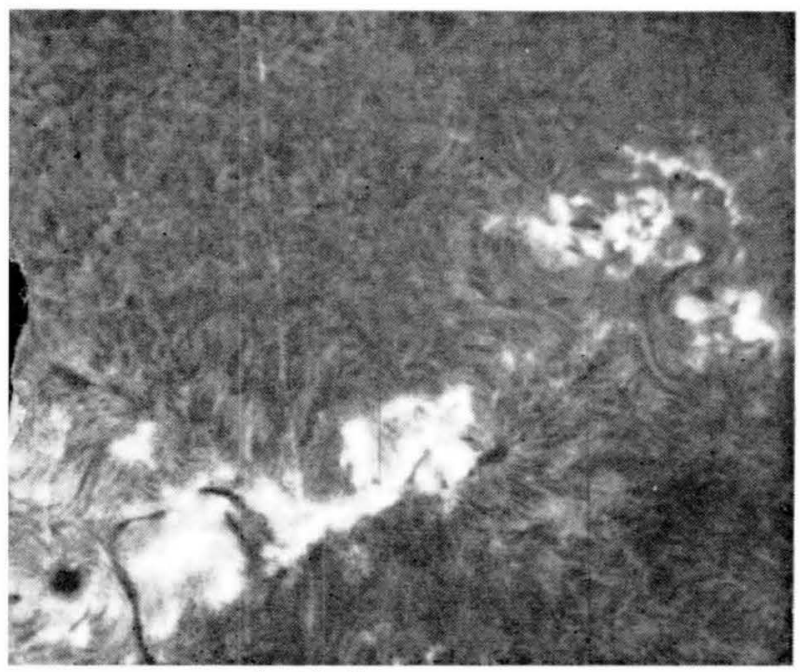

$9: 12: 04$

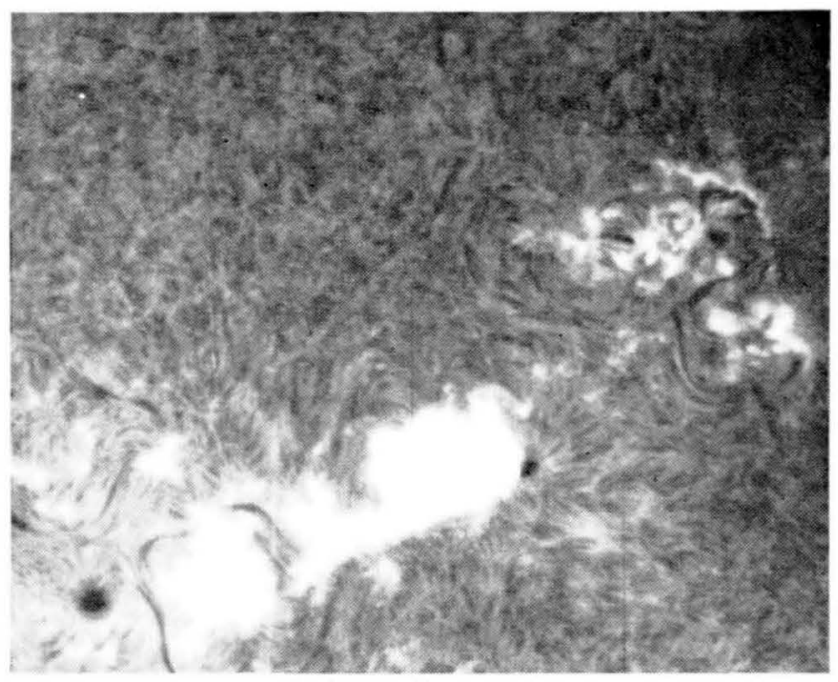

C
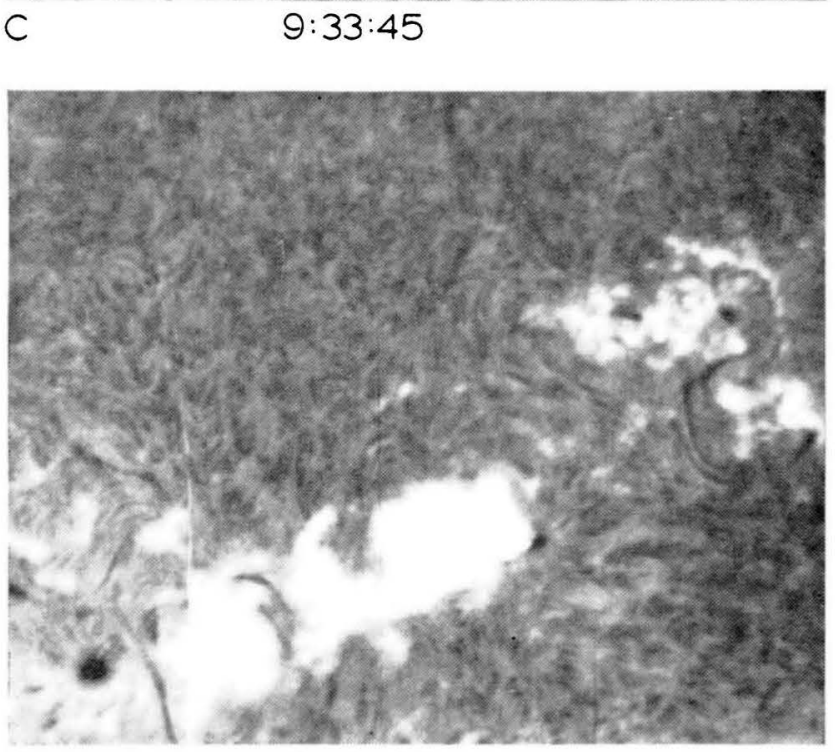

$9: 43: 40$

29 SEPTEMBER 1968

Fig. 7. Large fiare 29 September, photographed at Tel Aviv. After this flare, the $p$ spot moved rapidly away from the $f$ plage; its size decreased sharply.

The first and largest flare is seen on the Tel Aviv films to begin at 09:19:40 with the spreading of brightness northward from the $p$ spot. The X-ray flux measured by the University of California, Berkeley, OGO rose slowly starting 0912 UT, to a plateau at $0920 \mathrm{UT}$ of $900 \mathrm{cts} / \mathrm{sec}$, but there was no inkling that a great flare would occur. However, at 0929 UT, major brightening occurred simultaneously all over Plage 9687 , rapidly spreading from the neutral line to cover everything. At the same time, the hard X-ray flux rose rapidly to saturation. The third channel $(32-64 \mathrm{keV})$ did not saturate and peaked about 0937 UT, which is the time of maximum flare brightness. 
Although this channel decayed afterwards, the lowest channel (9-19 keV) showed a broad secondary maximum lasting until 0955 UT and a slow decay. This secondary maximum coincides with the period of slow spread to maximum area and development of a two-strand structure; one bright strand extended along the south edge of the whole region, all $p$ polarity; the other was a bright area covering the following spots.

This large flare was qualitatively different from the earlier large events in this region in that, although the flash phase was well marked, it lacked the explosive, dynamic appearance. There was no expulsion of material or bright wave of any sort. This may be connected with the declining nature of the region. It was much brighter, and covered a greater area than the flare on the 26th, although the X-ray fluxes were the same.

After this flare faded from view, another large flare occurred in the following part of the region, beginning $11: 13: 15$ in the $f$ polarity region just ahead of the large $f$ spot of $p$ polarity, and developing a second strand north of the large filament. Maximum was reached around 1121 UT. The OGO X-ray detector shows an X-ray event beginning 1113 UT and reaching a maximum of $5000 \mathrm{cts} / \mathrm{sec}$ in the $9-19 \mathrm{keV}$ channel.

After these flares, activity rapidly declined in 16997, although several more flares were reported. The next rotation, the center appeared as a weak plage without spots.

The most interesting aftermath of the flare on the 29th was the rapid separation of the $p$ spot from the neighboring plage, with development of a stable anti-plage (a term we use to denote a region of $p$ polarity around a sunspot without corresponding plage brightening) usually because the field is dominantly horizontal. This was already visible a few hours later.

\section{Discussion}

We have presented the development of this group as a special case with many typical features. The region was unusual in that the principal development took place in a very small sunspot with no apparent peculiarities. It looked so unimpressive that even on the 25th, I took large scale movies elsewhere on the disk. The region as a whole was somewhat unusual, with $p$ spots at both ends. The big stable spot at the west end of the center had appeared the previous rotation as Mount Wilson 16943. When it rotated off the disk 7 September, it showed no plages preceding. The large area of $f$ polarity plage preceding it apparently grew up on the backside of the sun. However, these did not play a role in the activity until the 29 th.

This large-scale polarity inversion with weak fields is not a typical circumstance of large activity; the fields must be strong, and the gradients steep.

The small-scale polarity inversion of 16997 is quite typical of the growth of inverted polarity near established spots. The spot 16997 is peculiar in that it is alone, with no nearby plage, leading the great, extended active region. The inverted polarity develops at a position angle about $70^{\circ}$, almost directly ahead of the spot. The rotation is very fast, the position angle being about $10^{\circ}$ on 25 September and $-70^{\circ}$ (or $300^{\circ}$ ) on the 26 th. The energy of rotation is, of course, as large as the volume we think is rotating. We should be fairly cautious about the rotation, since it may be the occurrence 
of new spots in successively rotated positions, which would be a different sort of phenomenon, no less interesting. In any event, we have found that $f$ polarity occurring in front of a spot (inverted polarity) migrates backwards, sooner or later, to the accompaniment of numerous flares.

The development of a dark anti-plage around the leading spot on the 29th and consequent stabilization is an interesting feature. Usually, the bright encroaching plage is $f$ polarity; the field lines must emerge vertically from the spot and turn downward. When a well-developed penumbra appears along with a 'whirlpool' structure, the field is dominantly horizontal. Small flares will occur in such a configuration only if bright plage appears near the penumbra. Rust (1968) has shown the role of small satellite spots in this situation.

The beautiful developments of 25 September show graphically the role of sunspot changes in flare production. The continuous loop formation as the spots grow shows that lines of force from the new spots are erupting through the atmosphere. The large flares during the first half of the 26th are clearly associated with the twinning and disappearance of the old $p$ spot and the appearance of the new one. The flares occur at the right times, and the first points to brighten are at the sunspots; motion is predominantly away from them. Feldman, Kozlovsky and Zirin (to be submitted to Solar Physics) found similar behavior in the spot group which transited the meridian 24 October 1968.

\section{Acknowledgements}

I wish to thank the observers on both Photoheliographs, and, particularly, Dr. Feldman for his supervision of the Tel Aviv Program. Dr. Howard kindly supplied the Mount Wilson data.

\section{Reference}

Rust, D.: 1968, in K. O. Kiepenheuer (ed.), 'Stucture and Development of Solar Active Regions', IAU Symp. 35, 77. 